\title{
Information Retrieval for Teaching Files: A Preliminary Study
}

John M. Bramble, Michael F. Insana, and Samuel J. Dwyer III

A computer algorithm for information retrieval from an electronic teaching file has been developed. This index enables the user to retrieve cases from a teaching file, based on the input of a combination of features. The algorithm is based on nearest neighbor analysis, and is programmed in the " $\mathrm{C}$ " language. A teaching file with this index is very easy to use as a reference resource for diagnosing unknown cases. A model was developed for a preliminary test of how likely a user would be to review a teaching file case that is the same diagnosis as an unknown case, thereby reducing uncertainty of diagnosis. The model used 110 cases of arthritis radiographs of hands scored by a skeletal radiologist. The result of the model suggests that the correct diagnosis would be reviewed $83 \%$ of the time. A standard method of reducing uncertainty of diagnosis (the maximum likelihood discriminant function) would have picked the correct diagnosis $78 \%$ of the time. The results indicate that a teaching file with the computer index is a practical tool for dealing with the uncertainty in diagnosis of unknown cases. The computer index could be included with videodisc-based teaching files (such as the American College of Radiology files). Using teaching files as a reference for interpreting unknown cases may reduce interobserver variability. (c) 1990 by W.B. Saunders Company.

KEY WORDS: arthritis, decision support, nearest neighbor analysis, information retrieval.

$\mathbf{T}$ HERE IS A VAST wealth of information available in modern medicine. Gaining access to this information is laborious. Computers can serve physicians by streamlining access to medical information.

Radiology teaching files are a prime example of an important source of information. Current methods of indexing and retrieving cases limit use of this information. If the practicing radiologist is confronted with a patient's study with radiographic findings that are difficult to interpret, it would be ideal to review cases with

From the Department of Diagnostic Radiology, University of Kansas Medical Center, Kansas City.

Address reprint requests to John $M$. Bramble, MD, Assistant Professor of Diagnostic Radiology and Chief of Musculoskeletal Radiology. Department of Diagnostic Radiology, 2-168 Bell Memorial Hospital, University of Kansas Medical Center, 39th St and Rainbow Blvd, Kansas City, KS 66103 .

( 1990 by W.B. Saunders Company.

0897-1889/90/0302-0001\$03.00/0 similar findings from the radiology teaching file. The computer can be programmed to retrieve cases from teaching files to reduce uncertainty in clinical problems. With recent advances in technology, electronic media can be used to store and distribute radiology teaching files. An example of an electronic teaching file that is currently being distributed is the American College of Radiology teaching file. It is logical to include a computerized index with these electronic teaching files that allows retrieval of cases for reduction of uncertainty in everyday practice.

Designing an information retrieval system (index) for reduction of uncertainty is a fairly new problem. Most algorithms for information retrieval are designed so that the user can retrieve a certain subject from a body of information. The design goal of our algorithm is to retrieve subjects (cases) not necessarily known by the user, based on user input concerning what observations are certain (the radiographic findings). The performance measure is the percentage of example cases retrieved that have the appropriate information. The final goal is that the process should be rapid and straightforward.

This article will describe a computer index used by a computerized textbook. ${ }^{1}$ The computerized textbook was designed to incorporate an electronic teaching file with the traditional material of a textbook. The index in the computerized textbook allows the user to recall cases from a teaching file based on individual features.

The problem of recalling information based on the input of a combination of features is equivalent to classification problems found in pattern recognition and expert systems. The "smart index" must select teaching file cases according to similarity to an unknown case (a discriminant function). The accuracy of the discriminant function can be tested by established means. ${ }^{2} \mathrm{~A}$ model of use of the smart index is developed with this study to provide an initial estimate of the probability that a user will review a case from the teaching file that is of the same diagnosis as an unknown case.

\section{MATERIALS AND METHODS}

Cases from a teaching file representing rheumatoid arthritis (21 cases), psoriatic arthritis (29 cases), gout (19 cases), 
inflammatory osteoarthritis (20 cases), and pyrophosphate arthropathy ( 20 cases) were retrieved. Cases were excluded if the age of the patient was less than 18 years. The cases were reviewed by a skeletal radiologist and scored for the presence or absence of a list of 20 radiographic features in the hand radiographs (Table 1). Cases were also scored for the presence or absence of joint destruction (erosions, ankylosis) in each of 23 joints. Radiographic features that indicated the incidental presence of primary osteoarthritis were excluded based on the judgment of the skeletal radiologist.

The radiographic features of the teaching file cases served as the data base used for indexing the cases. The algorithm is an implementation of nearest neighbor analysis in which the metric (measure of closeness) is based on similarity of radiographic features. Each test case is entered into the index as the presence or absence of specific radiographic features and/or joint involvement. The number of discrepancies between the test case and each case in the data base is tabulated. The test case and each case in the data base are matched. A discrepancy is defined as the presence of a finding in either the unknown case or the data base case that is not present in the case being matched to. The cases in the data base are then listed in order of fewest discrepancies first. Cases with 10 or more discrepancies are not listed. The limitation of cases by number of discrepancies is the equivalent of the Parzen window method of nearest neighbor analysis. $^{3}$

The algorithm is programmed in the " $\mathrm{C}$ " language and uses bit vector representation for data compression. The data compression does not affect the accuracy of the index, but increases the speed of computation by allowing the data base

Table 1. Features of Arthritis

\begin{tabular}{ll}
\hline \multicolumn{1}{c}{ Findings } & \multicolumn{1}{c}{ Joints } \\
\hline Fusiform swelling & Thumb IP \\
Lobulated swelling & Thumb MCP \\
Central erosions & First CMC \\
Marginal erosions & Common CMC \\
Extraarticular erosions & Midcarpal \\
Pressure erosions & Radiocarpal \\
Periostitis & Uinar styloid \\
Whiskering & Index MCP \\
Osteophytes & Index PIP \\
Overhanging edge & Index DIP \\
Enthesophytes & Long MCP \\
Ankylosis & Long PIP \\
CPPD & Long DIP \\
Symmetry & Ring DIP \\
Subluxation & Ring PIP \\
Fragmentation & Ring MCP \\
Soft tissue Ca & Little DIP \\
Subchondral cysts & Little PIP \\
Symmetrical loss & Little MCP \\
Asymmetrical loss & Radial-ulnar joint \\
Subchondral sclerosis & \\
Tuftal atrophy & - \\
Joint demineralization & \\
\hline & \\
\hline
\end{tabular}

Abbreviations: CPPD, calcium pyrophosphate dihydrate; IP, interphalangeal; MCP, metacarpophalangeal; CMC, carpometacarpal; PIP, proximal interphalangeal; DIP, distal interphalangeal. of radiographic features to be stored entirely in high-speed computer memory. All computations were completed using the Commodore Amiga computer (Commodore Business Machines, West Chester, PA).

For the purposes of the evaluation, each case was removed from the data base and used as an unknown test case. This was done to eliminate the perfect match that would have occurred if the test case had not been removed from the data base. The nearest neighbor analysis was then performed. A user of the index might not be expected to review all of the cases listed by the index. Because the cases listed first (with the fewest discrepancies) are most likely to be reviewed, a simple model of likeliness of review was used. Each case was assigned a point value as 10 minus the number of discrepancies. The resulting point value is greatest for the cases most likely to be reviewed. The result was scored as correct if the diagnostic class with the most points was the diagnosis listed for the teaching file case.

Comparison was made with a standard discriminant function based on maximum likelihood criteria from decision theory (Fig 1). For this algorithm, the discriminant variable is calculated with the sum of the logarithm base 10 of the Bayes' Rule estimate of a posteriori probabilities. ${ }^{3}$ Each observation is treated as independent, which is a common assumption for simplicity.

For the purposes of the comparison, each case in the data base was used as an unknown. The discriminant function based on the entire data base was then computed. Each case in the data base was then processed with the maximum likelihood discriminant function. If the disease with the largest sum of log Bayes' Rule coefficients corresponded with the disease listed as the diagnosis in the teaching file, the classification was scored as correct.

\section{RESULTS}

The linear discriminant function based on maximum likelihood with the assumption of independence was correct in 86 of 110 cases (78\%). The nearest neighbor discriminant function was correct in 91 of 110 cases $(83 \%)$. Between 1 and 2 seconds was required for the nearest neighbor analysis computation of one unknown case.

\section{DISCUSSION}

The results of this preliminary study indicate that the nearest neighbor algorithm would provide reasonable accuracy in the recall of cases. In this particular set of data, the nearest neighbor analysis performed comparably to the maximum likelihood discriminant function. However, this performance measure is not intended as a means of choosing between the nearest neighbor algorithm and maximum likelihood discriminant functions. The performance measure does suggest that accuracy comparable to a widely employed discriminant function may be expected. 


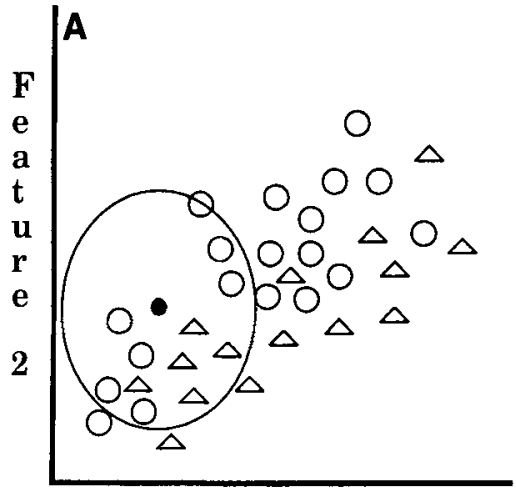

Feature 1

Nearest neighbor

discriminant function

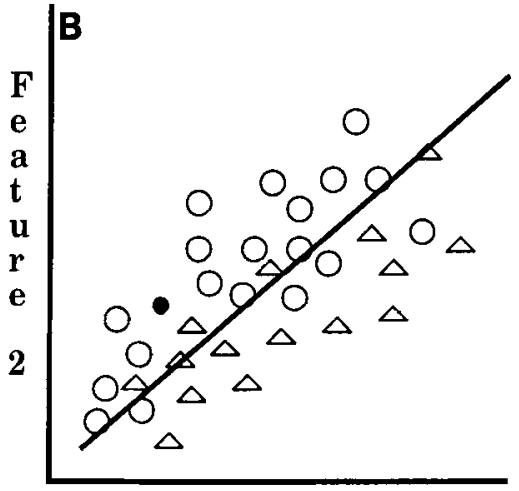

Feature 1

Maximum likelihood linear

discriminant function

Fig 1. Graphic comparison of nearest neighbor (NN) discriminant function with maximum likelihood (ML) discriminant function. An unknown case (black dot) has certain features represented by its position in feature space. Multiple teaching file cases from two diseases (represented by open circles and triangles) are also graphed according to the scores on two features. (A) The NN algorithm will retrieve all those cases lying within the large open circle. (B) The ML algorithm will retrieve all those cases on the same side of the decision boundary (diagonal line) as the unknown case.

The main reason for choice of the nearest neighbor algorithm over other methods of discriminant functions is the emphasis on similarity of findings. Review of cases with similar findings is important to provide feedback to the observer about accuracy of observations. Review of cases based on similar diagnosis will often present a variety of findings, not necessarily providing feedback to the observer about provisional findings in an unknown case.

One source of error in using the nearest neighbor algorithm is lack of a sufficient spectrum of cases in the teaching file. However, with the increasing use of digital images in radiology, the development of large teaching files becomes much more feasible. Another important source of error that was not evaluated is observer variability. Both interobserver and intraobserver variance in identification of features reduces the accuracy of any discriminant function. With a teaching file, at least there is immediate feedback on radiographic features that could reduce observer variability. The inaccuracy of observations by users has been a major problem for decision support systems developed as computer-aided diagnosis. ${ }^{4}$

The user's differential diagnosis of an unknown case can be affected by the diagnoses of the teaching file cases. However, probability of disease cannot be directly inferred by this approach. In the future, if the daily clinical load is made accessible by high-speed electronic storage
(PACS), this may be used to provide local estimates of probability of disease.

The similarity of an algorithm for information retrieval to techniques of decision analysis and expert systems is not by chance. The ultimate goal of both is to provide the user with information that is pertinent and can reduce the uncertainty (decision support). ${ }^{5}$ Any technique of decision analysis or expert systems could be used to develop a smart index that would reduce the number of cases that must be looked-up before the pertinent information is found. The nearest neighbor analysis algorithm seems especially suitable because of the ability to recall cases on the basis of similarity of radiographic features. The emphasis of the computerized index is slightly different from prior implementations of decision analysis or expert systems in the form of computer-aided diagnosis. Computer-aided diagnosis provided either statistics or critique that helped the physician make a decision. The computerized index provides a list of teaching file cases that, after review, are likely to help the physician make the correct decision. If interobserver variability is decreased by the information retrieval system, computer-aided diagnosis may become more accurate in general use.

The computer system IMAGE/ICON ${ }^{6}$ was developed as an expert system that critiques users' interpretations of radiographic features in the chest roentgenograms of patients with lym- 
phoreticular disorders. A teaching file of cases has been included with the IMAGE/ICON system to provide radiographic examples of abnormalities being discussed in the critique. The images are accessed with several indexes tied into the critique. The "size/extent" axis provides retrieval of cases based on radiographic findings with various permutations. The differential diagnosis axis retrieves cases with the selection criteria of probability of disease, similar to the maximum likelihood classifier. The investigators report that they are in the process of testing the accuracy of the multiple indexes.

Expert systems differ from methods of pattern recognition in that they tend to be symbolic processors, working with a knowledge base, as opposed to a data base. In the computerized textbook, no critique of users' decisions is provided because of the lack of symbolic processing. However, an expert's discussion of each individual case can be displayed with the images in the computerized textbook. The expert's discussion can provide additional information such as disease incidence, and variations from the general features categories (such as the presence of fine nodules in certain joint diseases) that could be useful to the user.

Standard relational data bases could be used for developing indexes to teaching files. However, the use of available data base programs is often difficult for recall of combinations of features (requiring the use of a data base query lan- guage). Furthermore, most data bases do not provide a mechanism for recalling cases that are not perfect matches to a combination of features. Because there seldom is a perfect match of features, the algorithm for recall of cases must deal with the uncertainty of less than perfect matches. With the nearest neighbor algorithm, the best matches are listed first, followed in descending order by additional examples with the variations in age, sex, and additional radiographic features (Fig 2). The combination of pattern recognition techniques with data base functions has been termed an extended data base. Extended data bases are more suitable for scientific data than standard relational data bases. $^{?}$

Hypertext provides an alternate approach to access of information. ${ }^{8}$ Hypertext may actually be considered a combination of a graphic user interface with a data base model. Different versions of hypertext contain similar user interfaces with significantly different data base models. The most common data base model is that of linked frames (of text, or graphics, or sound). Most versions of hypertext do not provide the ability to access information based on combinations of features. Furthermore, hypertext methods do not allow the retrieval of those cases that are near matches to an unknown case (dealing with uncertainty). The method of nearest neighbor analysis (with an appropriate data base model) could be added to hypertext systems as an
Fig 2. The smart index is used to retrieve cases with the most similarity to the findings of an unknown case. Each case can then be retrieved from either conventional or electronic storage.

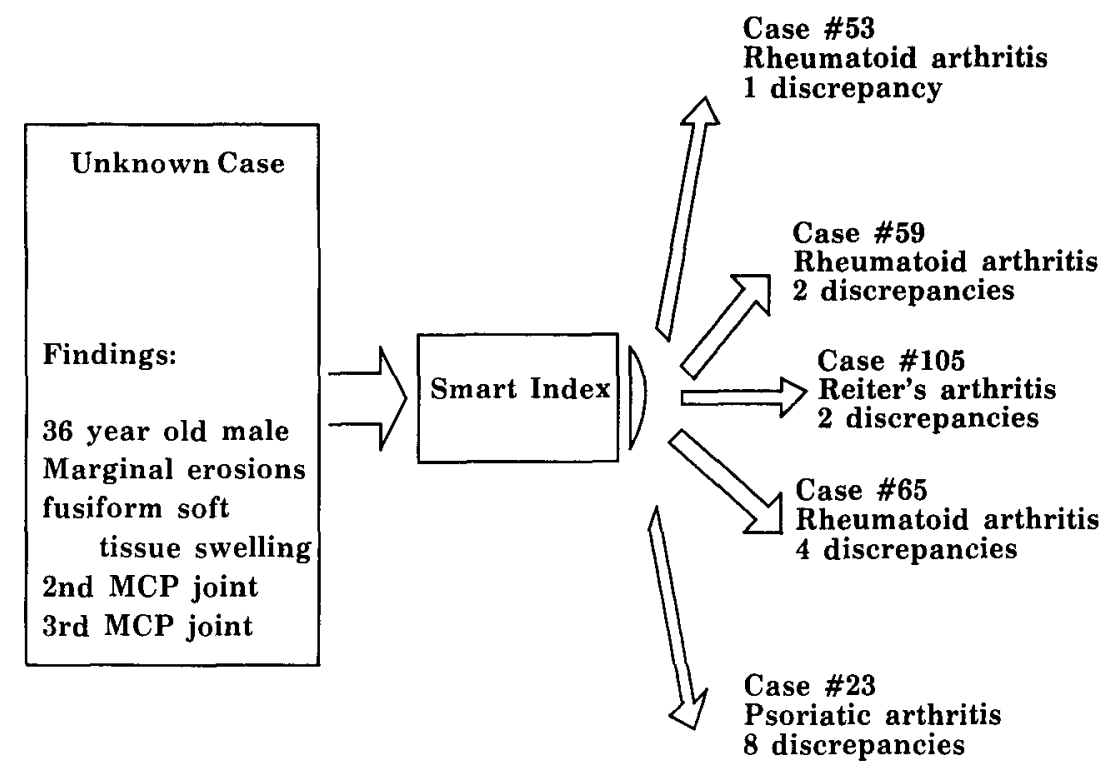


index to provide combinatorial and uncertainty methods. The next generation of hypertext software is likely to incorporate at least the relational data base model, while retaining the same user interface.

Relational data bases, hypertext, and the index described in this article all represent information retrieval systems. The measure of performance we have made is similar to performance measures derived in information retrieval theory. ${ }^{9}$ Probability of relevance in information retrieval theory is defined as the probability that a retrieved document is relevant to the user. The measure of performance we have used departs from information retrieval theory in that the medical user is uncertain about the relevance of the retrieved information. The uncertainty justifies the use of performance measures developed for computer-aided diagnosis.

An advantage to the index in the computerized textbook is that no programming is required to develop and use the index (as opposed to relational data bases and hypertext). All that is required of the person assembling the index is the entry of the radiographic findings for each case, as well as a discussion of the case. The entry of radiographic findings may be accomplished by a graphic user interface or processing of dictated reports (if the grammar of the report is simple).

An unresolved issue that may prevent implementation of the nearest neighbor analysis is the length of time for encoding cases and ease of use to the end user. In the authors own experience, encoding each individual case required roughly two to three times the length of time for an ordinary dictation. Encoding each case was accomplished by recording findings on a form (pen and paper). The cases were then transferred to the computer with a graphical user interface. Each positive finding is selected with a mouse pointer. The location of disease is also selected with a mouse pointer. The author is an experienced computer user and the time for encoding will likely increase for those with less experience. The advantage of coding compared with dictation is that the information tends to be more complete.

Arthritis is a subject that requires information on a large number of radiographic findings in a large number of locations. Other subjects may require a lesser number of features for findings or location, such as differential diagnosis of interstitial lung disease. This approach may also be useful for subjects not considered for differential diagnosis. A simple example would be magnetic resonance imaging of the knee for internal derangement. Multiple cases in a teaching file with normal variants, pitfalls in diagnosis, and varieties of pathology could be used to compare and confirm identification of features in an unknown case. This would be a very helpful tool for those unfamiliar with this technique. Expert's discussion of these teaching file cases can help educate radiologists on the clinical ramifications of the observations.

With the capabilities provided by an index based on the nearest neighbor analysis, a large teaching file becomes a very effective reference for clinical practice. As programmed in this implementation, it would also be easy to update the teaching file with new cases as they become available. Teaching files could be accumulated by publication of case series with specific information about what features are present in each individual case. The system can also be integrated with radiographic report generation methods (voice recognition or coding). Clinical cases that are coded for the purposes of report generation can also be indexed by this method. ${ }^{10}$ Teaching files would then include the less common cases ordinarily found in teaching files, as well as the cases representative of the local clinical population (Fig 3).

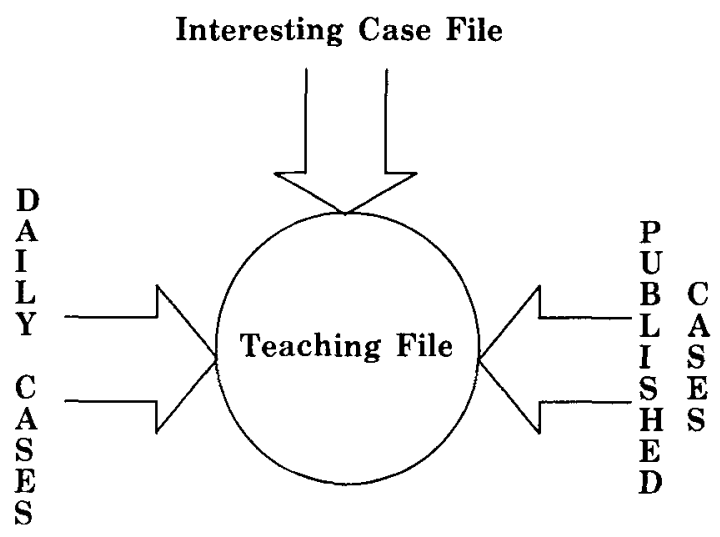

Fig 3. With the nearest neighbor index, the teaching file can begin with current interesting case files. New cases can be included from published case reports and daily cases. 


\section{CONCLUSION}

An index to teaching files can be developed, which helps users to recall cases that have similarities to unknown cases. This information retrieval system makes it more practical to use teaching files as reference material. The ability of an index to recall cases that are appropriate is a discriminant function that can be tested. A model for preliminary study using the nearest neighbor algorithm was tested. The results indicate that reasonable accuracy of recall of pertinent cases can be expected with use of the nearest neighbor algorithm. Furthermore, cases can be retrieved based on observations by the user. The goals of information retrieval from a teaching file can be met by use of the nearest neighbor algorithm. Retrieval of cases from a teaching file based on observation of findings may help reduce interobserver variability. Ultimately, the accuracy of the method could be tested when an electronic teaching file of images becomes available.

\section{ACKNOWLEDGMENT}

The authors wish to thank Dr Donald Resnick for the use of his teaching file as a source for the cases used in this study.

\section{REFERENCES}

1. Bramble JM, Resnick DL, Murphey MD, et al: Computer-aided diagnosis as an educational tool. Presented at the 74th Scientific Assembly and Annual Meeting of the Radiological Society of North America, Chicago, IL, November 1988

2. Fukunaga K: Intro to Statistical Pattern Recognition. New York, NY, Academic, 1972, p 149

3. Duda RO, Hart PE: Pattern Classification and Scene Analysis. New York, NY, Wiley, 1973, pp 31-32, 88-90

4. Piraino DW, Richmond BJ, Uetani M, et al: Problems in applying expert system technology to radiographic image interpretation. J Digital Imaging 2:21-26, 1989

5. Lusted IB: Introduction to medical decision making. Springfield, IL, Thomas, 1968, $\mathrm{p}$ vii

6. Swett HA, Fisher PR, Cohn AI, et al: Expert systemcontrolled image display. Radiology 172:487-493, 1989
7. Martin D: Advanced Database Techniques. Cambridge, MA, MIT, 1986, p 179

8. Jaffe CC, Lynch PJ, Smeulders AW: Hypermedia techniques of diagnostic imaging instruction: Videodisk echocardiography encyclopedia. Radiology 171:4475-4480, 1989

9. Campagnoni FR, Ehrlich K: Information retrieval using a hypertext-based help system, in Belkin NJ, van Rijsbergen CJ (eds): Proceedings of the Twelfth Annual International ACMSIGIR Conference on Research and Development in Information Retrieval. New York, NY, ACM, 1989, pp 212-220

10. Bramble JM, Chang CHJ, Martin NL: A reportcoding system for integration into a digital radiology department. AJR 152:1109-1112, 1989 